Peter R. Kornaat Margreet Kloppenburg

Ruby Sharma

Stella A. Botha-Scheepers

Marie-Pierre Hellio Le Graverand

L. Napoleon J. E. M. Coene

Johan L. Bloem

Iain Watt

\section{Bone marrow edema-like lesions change in volume in the majority of patients with osteoarthritis; associations with clinical features}

Received: 6 January 2007

Revised: 3 June 2007

Accepted: 5 June 2007

Published online: 7 September 2007

(C) Springer-Verlag 2007
P. R. Kornaat $(\bowtie) \cdot$ R. Sharma

J. L. Bloem · I. Watt

Department of Radiology,

Leiden University Medical Center,

Albinusdreef 2

2333 ZA Leiden, The Netherlands

e-mail: P.R.Kornaat@lumc.nl

Tel.: +31-71-5262052

Fax: +31-71-5248256

M. Kloppenburg

S. A. Botha-Scheepers

Department of Rheumatology,

Leiden University Medical Center,

Leiden, Netherlands
M.-P. H. Le Graverand

Pfizer Groton,

Groton, CT, USA

L. N. J. E. M. Coene

Department of Orthopaedic Surgery,

Haga Hospital,

The Hague, Netherlands

\begin{abstract}
It has been suggested that bone marrow edema-like (BME) lesions in the knee are associated with progression of osteoarthritis (OA). The purpose of our study in patients with OA was to evaluate prospectively changes of BME lesions over 2 years and their relationship with clinical features. Magnetic resonance (MR) images of the knee were obtained from 182 patients (20\% male; aged 4376 years; mean age 59 years) who had been diagnosed with familial symptomatic OA at multiple joint sites. MR images were made at baseline and at 2 years follow-up. BME lesions in 2 years were associated with clinical
\end{abstract}

features assessed by Western Ontario and McMaster Universities Osteoarthritis (WOMAC) scores. A total of $327 \mathrm{BME}$ lesions were recorded. Total size of BME lesions changed in 90 patients $(66 \%)$. Size of individual lesions changed in 147 foci $(45 \%)$ : new lesions appeared in 69 (21\%), existing lesions disappeared in 32 $(10 \%)$, increased in size in $26(8 \%)$ and decreased in size in $20(6 \%)$ lesions. Increase or decrease of BME lesions, over a 2-year time period, was not associated with severity of WOMAC scores. BME lesions fluctuated in the majority of patients with OA over a 2-year time period. These changes were not associated with severity of WOMAC scores at the study end point.

Keywords Knee $\cdot$ MRI $\cdot$ BME $\cdot$ WOMAC $\cdot \mathrm{OA}$

\section{Introduction}

Knee osteoarthritis (OA) is a chronic, progressive joint disease, leading to pain and loss of function in a considerable proportion of patients, with great impact and consequences in the ageing population of the industrialized world. Disease markers need to be identified in order to predict and quantify progression. One possible marker in $\mathrm{OA}$ is the so-called bone marrow edema-like (BME) lesions [1].

Unfortunately, the role of $\mathrm{BME}$ in $\mathrm{OA}$ remains controversial, as contradictory results have been reported. BME detected with magnetic resonance (MR) imaging has been associated with clinical symptoms in patients with 
OA $[2,3]$. However, other studies reported no association between BME lesions and clinical symptoms [4-6]. Further, the role of BME as a marker for progression of $\mathrm{OA}$ is open to discussion. In a study by Felson et al. [7], $\mathrm{BME}$ was associated with progression of $\mathrm{OA}$ as assessed by joint space narrowing on conventional radiographs. On the other hand, in a study by Phan et al. [5], changes in BME did not significantly change with progression of disease assessed by Western Ontario and McMaster Universities Osteoarthritis (WOMAC) scores.

Since these contradictory results regarding the association between BME and clinical features have been reported, the purpose of our study was to evaluate changes in BME lesions over a 2-year period, and associate them with clinical features.

\section{Patients and methods}

Patients

The present prospective study is part of the ongoing GARP (Genetics, Osteoarthritis and Progression) study [8]. The primary goal of the GARP study is the identification of genetic susceptibility determinants to $\mathrm{OA}$ and disease progression in middle-aged sibling pairs with $\mathrm{OA}$ at multiple joint sites. MR image sets of the knee were obtained in 182 patients at study entry and after 2 years [6]. Only one knee, the most symptomatic, was imaged per patient. In the present, study $39 \%(71 / 182)$ of the patients had symptomatic knee OA in their imaged knee, defined as pain or stiffness on most days in the month prior to study entry, and osteophytes on radiographs. As the purpose of the MR study was to assess progression of OA, no images were made of a knee that already had a maximum Kellgren and Lawrence score of grade 4 [9].

\section{Clinical assessment}

Clinical data were assessed by WOMAC to assess pain, stiffness and functional impairment of the imaged knee at the 2-year time point, not at baseline [10].

\section{MR acquisition}

Knees were imaged using a transmit-receive four-channel knee coil in a 1.5-T superconducting magnet (Philips Medical Systems, Best, the Netherlands). Each examination consisted of: coronal proton density and T2-weighted dual spin echo (SE) images (with repetition time (TR) of $2,200 \mathrm{~ms}$; echo time (TE) of $20 / 80 \mathrm{~ms}$; number of excitations per data line (NEX) 2; $5 \mathrm{~mm}$ slice thickness; $0.5 \mathrm{~mm}$ intersection gap; $160 \mathrm{~mm}$ field of view (FOV);
$256 \times 205$ acquisition matrix, 18 slices); sagittal proton density and T2-weighted dual SE images (TR 2,200 ms; TE 20/80 ms; NEX 2; $4 \mathrm{~mm}$ slice thickness; $0.4 \mathrm{~mm}$ intersection gap; $160 \mathrm{~mm}$ (FOV); $256 \times 205$ acquisition matrix, 20 slices); sagittal 3D T1-weighted spoiled gradient echo (GE) frequency selective fat suppressed images (TR $46 \mathrm{~ms}$; TE $2.5 \mathrm{~ms}$; NEX 1; flip angle $40^{\circ} ; 3.0 \mathrm{~mm}$ slice thickness; slice overlap $1.5 \mathrm{~mm}$; no gap; $180 \mathrm{~mm}$ (FOV); 256 acquisition matrix, 80 slices); and axial proton density and T2-weighted turbo spin echo (TSE) fat suppressed images (TR 2,500 ms; TE 7.1/40 ms; NEX 2; 2 mm slice thickness; no gap; $180 \mathrm{~mm}$ (FOV); 256 acquisition matrix, 62 slices). Total acquisition time (including the initial survey sequence) was $30 \mathrm{~min}$.

\section{MR interpretation}

All MR images were analyzed in known chronological order by means of consensus between three experienced readers, using a comprehensive score form [11]. During the assessment, the readers were blinded to radiographic results, patient symptoms and patient age. In cases of disagreement between the readers the more conservative, less severe score was recorded. BME or cysic lesions was assigned to any one or more of the following anatomic locations: the crista patellae, medial or lateral patellar facets, the medial or lateral trochlear articular facets, the medial or lateral femoral condyles, the medial or lateral tibial plateaux.

BME lesions were defined as an ill-defined area of increased signal intensity on $\mathrm{T} 2$-weighted images in the subchondral bone, extending away from the articular surface over a variable distance [12]. The lesions were graded as follows: grade 0 , absent; grade 1, minimal (diameter $<5 \mathrm{~mm}$ ); grade 2, moderate (diameter $5 \mathrm{~mm}-$ $20 \mathrm{~mm}$ ); grade 3 , severe (diameter $>20 \mathrm{~mm}$ ). The maximum two-dimensional diameter was measured to grade the lesion. A total BME score of the knee was calculated by adding all grades of each BME lesion in the knee. Maximum possible knee score was 27 (grade 3 times nine anatomic locations). In the total study a maximum of 1,638 BME lesions (182 patients times nine anatomic locations) could be scored.

Subchondral cysts were defined as well-defined foci of high signal intensity, with low signal intensity margins, on T2-weighted images, in the bone underlying the joint cartilage. Their greatest dimension was measured and they were graded as follows: grade 0 , absent; grade 1, minimal ( $<3 \mathrm{~mm})$; grade 2 , moderate $(3-5 \mathrm{~mm})$; grade 3 , severe $(>5 \mathrm{~mm})$. A total score of the knee was calculated by adding all grades of each cystic lesion in the knee. Maximum possible knee score was 27 (grade 3 times nine anatomic locations). In the total study a maximum of 1638 cystic lesions (182 patients times nine anatomic locations) could be scored. 
Table 1 Patient characteristics $(n=182)$

\begin{tabular}{ll}
\hline & At baseline \\
\hline Age years, median (range) & $59(43-76)$ \\
Female sex, $(\%)$ & $157(80 \%)$ \\
Body mass index $\left(\mathrm{kg} / \mathrm{m}^{2}\right)$, median (range) & $25.7(20.2-40.0)$ \\
Symptomatic knee OA, $n(\%)^{\mathrm{a}}$ & $71(39 \%)$ \\
Kellgren \& Lawrence Score 0/1/2/3/4, No. & $59 / 53 / 60 / 10 / 0$ \\
& Over 2 years \\
Bone marrow edema-like lesions, $n(\%)^{\mathrm{b}}$ & $128(70 \%)$ \\
Grade 0/1/2/3 $^{\mathrm{c}}$ & $54 / 56 / 62 / 10$ \\
Cysts, $n(\%)^{\mathrm{b}}$ & $100(55 \%)$ \\
Grade 0/1/2/3 & $82 / 60 / 37 / 3$ \\
& At 2 years \\
WOMAC Pain scores, median (range) $^{\mathrm{d}}$ & $13(0-99)$ \\
WOMAC Stiffness scores, median (range $^{\mathrm{d}}$ & $18(0-99)$ \\
WOMAC Function scores, median (range) $^{\mathrm{d}}$ & $14(0-98)$ \\
\hline
\end{tabular}

${ }^{a}$ Defined as pain or stiffness on most days of the month prior to study entry, in combination with osteophytes on radiographs

${ }^{\mathrm{b}}$ During 2 years

${ }^{c}$ Maximal grade per patient

${ }^{\mathrm{d}} n=157$

\section{Statistical analysis}

Odds ratios (ORs) with 99\% confidence intervals (CIs) were used to show any association between BME size changes with cystic size changes.

The difference in WOMAC scores between patients without BME lesions (group A) and patients with BME lesions (group B: unchanged BME lesions over 2 years; group $\mathrm{C}$ : increasing size of $\mathrm{BME}$ lesions over 2 years; group D: decreasing size of BME lesions over 2 years) was calculated by linear mixed models in SPSS for Windows, version 12.0 (SPSS, Chicago, Ill.) with a random intercept to adjust for the familial effect within sib pairs. Adjustments were made for age, sex and body mass index (BMI). Estimates of fixed effects were reported with $95 \%$ CIs.

\section{Results}

In total 182 patients were monitored over a period of 2 years (Table 1). Forty-six (25\%) patients did not have BME lesions; thus, 136 patients $(75 \%)$ had one or more BME lesions at any time point. In $46(34 \%)$ of patients, BME scores did not fluctuate, whereas they changed in the other $90(66 \%)$ patients. The total BME score per individual patient increased in $54(40 \%)$ patients. It decreased in 27 patients $(20 \%)$, and total BME score remained unchanged in nine $(7 \%)$ patients. Individual BME scores did change in this last group without resulting in a change of the total BME score. A total of 327 BME lesions were detected from a possible total of 1,638 lesions (Table 2). One hundred and forty-seven (45\%) BME lesions changed: 69 new lesions appeared on the second MR (21\%), $26(8 \%)$ increased, $20(6 \%)$ decreased in size, and 32 $(10 \%)$ were no longer visible on the second MR scan (Fig. 1). It was noted that more lesions appeared or increased than decreased or disappeared.

One hundred $(55 \%)$ patients had one or more cystic lesions at any time. In 44 patients (44\%), total cystic score did not fluctuate over time, whereas in the other 56 patients $(56 \%)$ the total cystic score changed in size. In 36 of these 56 patients $(64 \%)$ the cystic score increased, it decreased in 18 patients $(32 \%)$, and the total cystic score remained unchanged irrespective of changes on the level of individual cysts in two patients (4\%). A total of 222 cystic lesions were detected from a possible total of 1,638 sites (Table 2). Seventy cystic lesions (32\%) changed: 32 new cysts appeared on the second MR (46\%), $14(20 \%)$ increased, six (8\%) decreased in size, and $18(26 \%)$ were no longer visible on the second MR (Fig. 2).

Cystic and BME lesions were both present in the same anatomic location (associated lesions) in 191 cases. A change of BME lesions or cystic lesions was associated with a change in size of an adjacent cystic or BME lesion (OR: 6.2; 95\% CI: 3.2-12.3). In 47 cases both BME lesions and cystic lesions changed. When cystic and BME lesions were both present in the same anatomic location, size changes (increase or decrease) were in same direction (OR: 37; 95\% CI: 6-210) (Table 2).

One hundred and fifty-seven (86\%) patients completed a WOMAC questionnaire of the imaged knee at 2 years. The WOMAC pain and function scores for patients without and with BME lesions over 2 years are shown in Fig. 3a and b, respectively. The mean WOMAC scores did not differ between the different patient groups; even when BME lesions completely disappeared, lower WOMAC scores were not recorded. The mean difference in WOMAC pain

Table 2 Cystic and BME lesions per patient changing over 2 years in 182 patients $(n=1,638)$

\begin{tabular}{|c|c|c|c|c|c|c|}
\hline & & \multicolumn{5}{|c|}{ BME lesions } \\
\hline & & No BME & No change & Increase & Decrease & Total \\
\hline \multirow[t]{5}{*}{ Cysts } & No cyst & 1,280 & 77 & 38 & 21 & 1,416 \\
\hline & No change & 24 & 87 & 24 & 17 & 152 \\
\hline & Increase & 2 & 11 & 30 & 3 & 46 \\
\hline & Decrease & 5 & 5 & 3 & 11 & 24 \\
\hline & Totals & 1,311 & 180 & 95 & 52 & 1,638 \\
\hline
\end{tabular}


Fig. 1 a-d Axial T2-weighted turbo spin echo fat suppressed images. Increase (a at baseline, marrow edema-like lesions over 2 years at the crista patella oral condyle (arrowhead). Decrease (c at baseline, $\mathbf{d}$ after two years) in size of bone marrow edema-like lesions over 2 years at the crista and medial part of the patella (open arrowhead) b after two years) in size of bone (arrow) and at the medial fem-

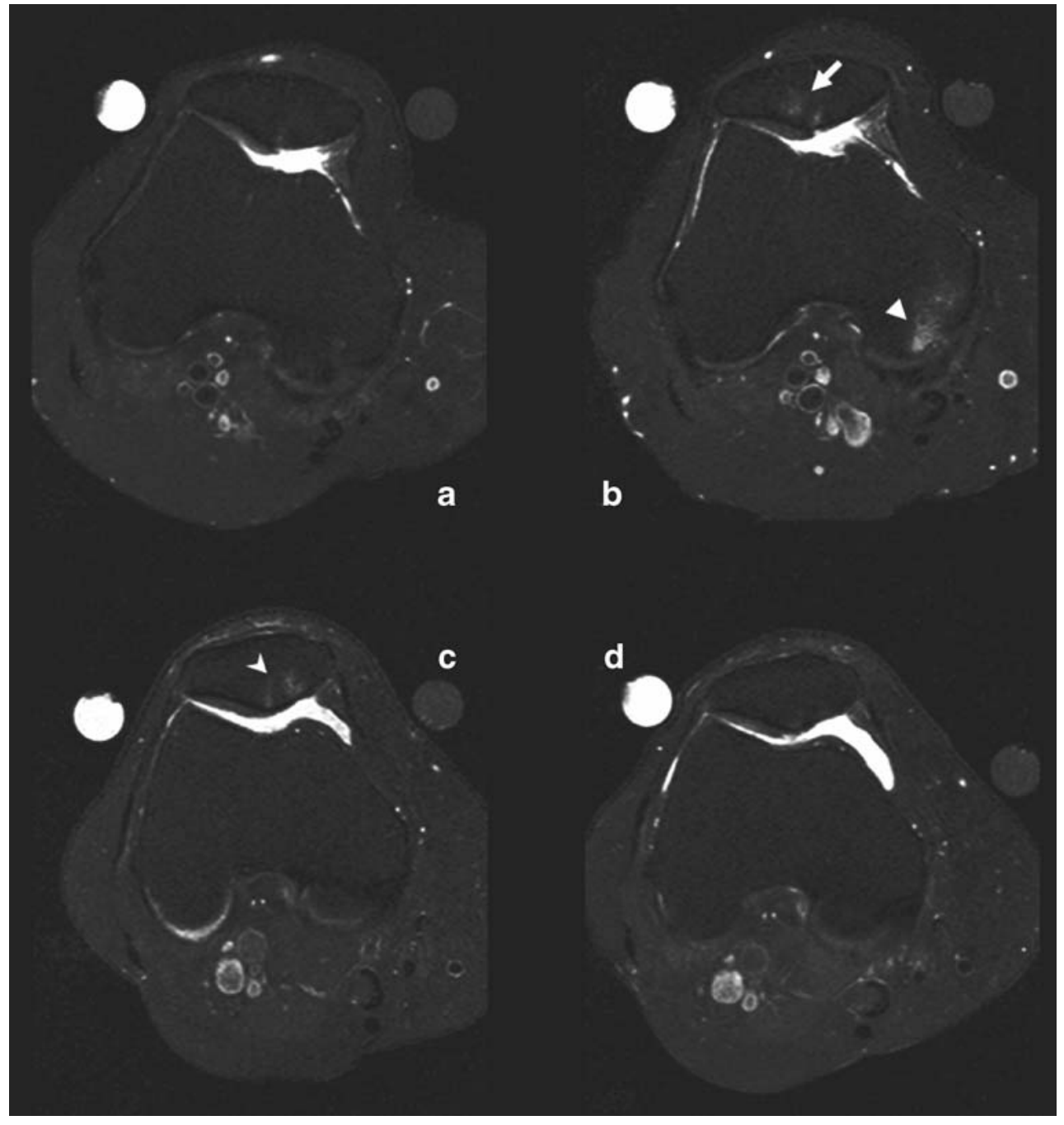

Fig. 2a, b Axial T2-weighted turbo spin echo fat suppressed images. Disappearing cyst at the lateral femoral condyle (arrow). a At baseline, $\mathbf{b}$ after 2 years
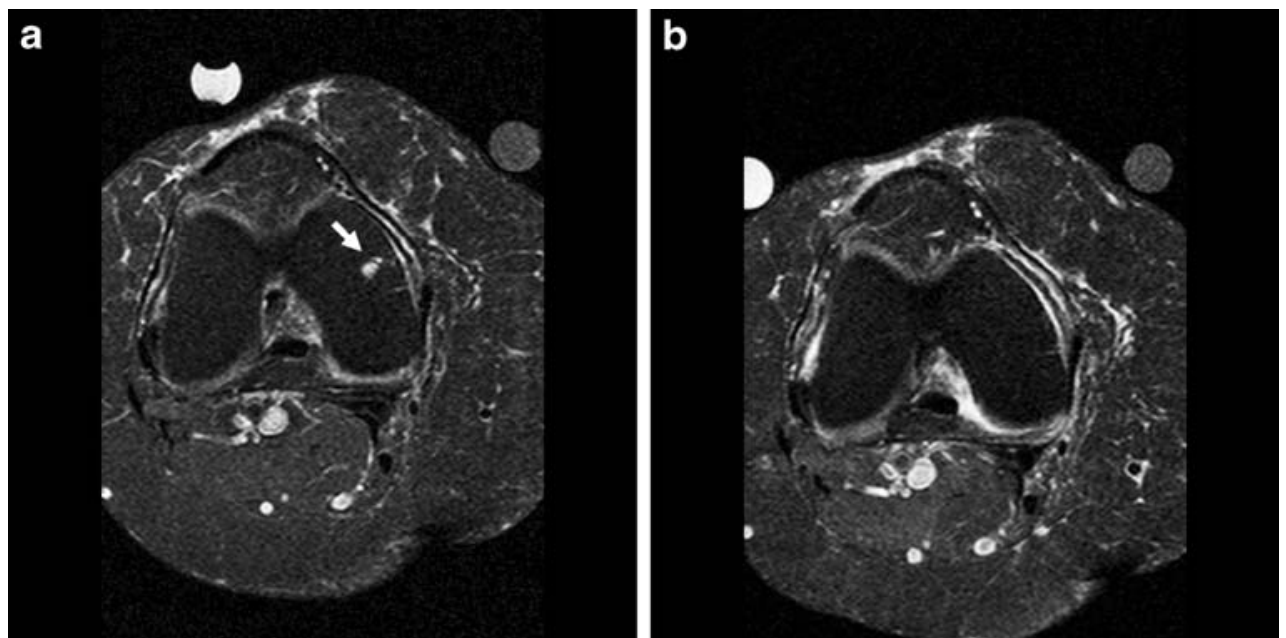
scores between patients with unchanged BME lesions, with increasing BME lesions and with decreasing BME lesions compared with patients without BME lesions were $2(95 \%$ CI-8 to 12$), 2$ (95\% CI-8 to 11$)$ and 1 (95\% CI-11 to 12$)$ respectively (Fig. 3a). The mean difference in WOMAC function scores between patients with unchanged BME lesions, with increasing BME lesions and with decreasing BME lesions compared to patients without BME lesions were -2 (95CI-12 to 8 ), -4 (95CI-13 to 6 ) and -4 (95CI-15 to 8 ) respectively (Fig. $3 b$ ).
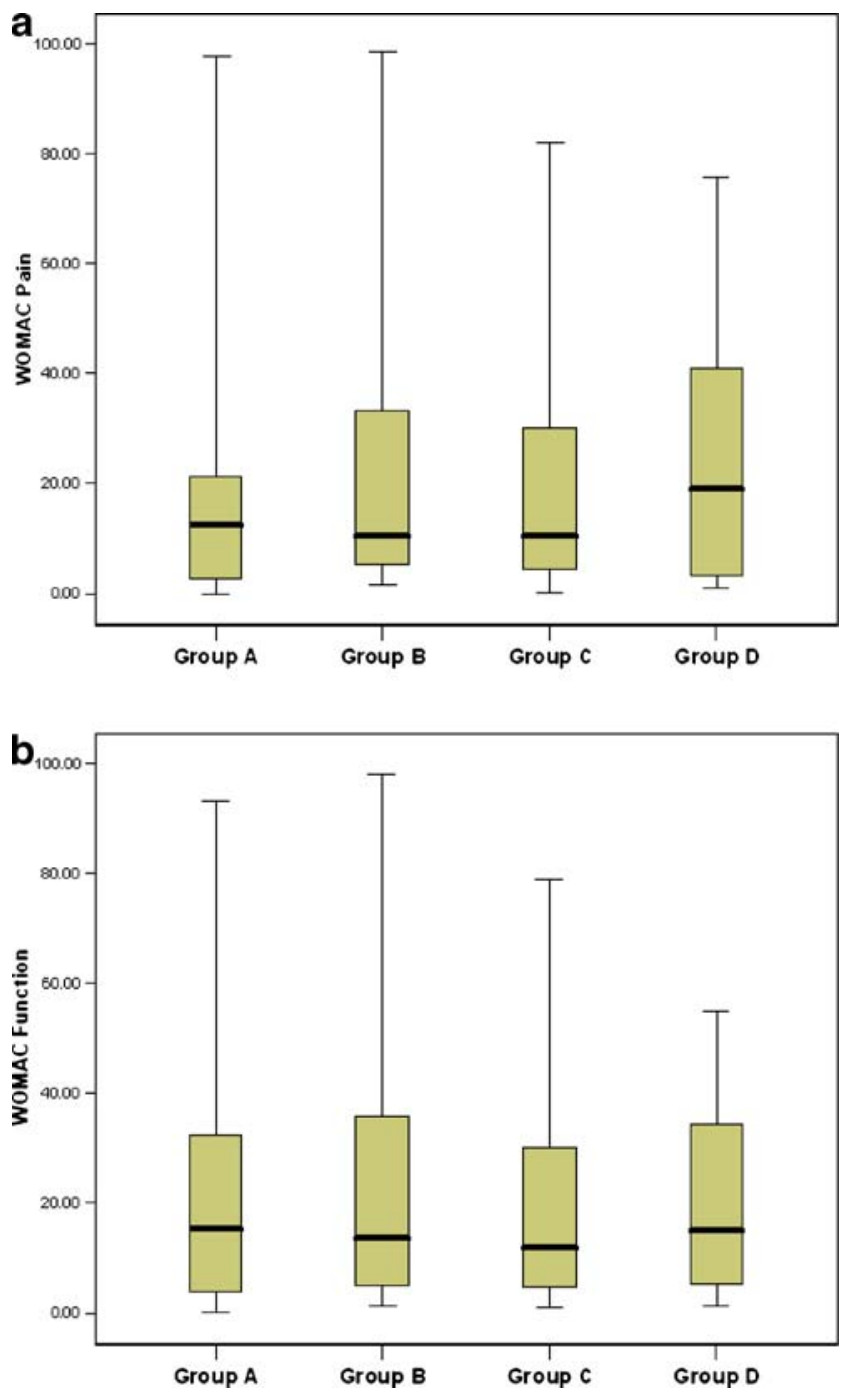

Fig. 3 Association between WOMAC pain (a) or function (b) scores and bone marrow edema-like lesions. Group A: patients with no bone marrow edema-like lesions; Group B: patients in whom bone marrow edema-like lesions did not change over 2 years; Group $C$ : patients in whom bone marrow edema-like lesions increased in size over 2 years; Group D: patients in whom bone marrow edemalike lesions decreased in size over 2 years. Box plots show the median, interquartile range, minimum and maximum values

\section{Discussion}

The majority (75\%) of patients with familial OA at multiple sites have BME lesions visualized when two sequential MR scans are made with a 2-year time interval. In the majority $(66 \%)$ of these patients with BME lesions, the total size of BME changed over this 2-year time period. Our study also demonstrates that cysts and BME fluctuations are associated. However, no association existed between changes in BME lesions and severity of WOMAC scores after 2 years.

The finding, that BME lesions fluctuate in $66 \%$ of the patients, indicates that BME is part of a dynamic process in OA. BME is not a constant finding, as opposed to hyaline cartilage loss for example. Thus, it is important to realize that the finding of BME lesions in patients with OA represents only a single snapshot in time. The variability of $\mathrm{BME}$ lesions has been noted before [5]. It is also interesting and important to note that $10 \%$ of BME lesions disappear completely. This is particularly noteworthy if BME is to be used as an inclusion criterion, outcome parameter or surrogate endpoint in drug trials or clinical outcome studies.

The second finding of the present study is that when cystic lesions and BME lesions are in close proximity; the direction in which they change is identical. This is an interesting finding as the role of cysts in $\mathrm{OA}$ is unclear. Their exact pathophysiology is uncertain, as is their prognostic significance. A recent study by Carrino et al. [13] also showed a change in cyst size was accompanied by a change in oedema-like signal size. That study also showed subchondral cysts developing in pre-existing regions of subchondral bone marrow oedema-like signal.

The third finding is that changes in BME lesions did not correlate with severity of WOMAC scores. Patients in whom BME increased did not have a higher WOMAC score than patients with a decrease in BME size. Even when BME completely disappeared, lower WOMAC scores were not recorded. Previous work has demonstrated that pain, as assessed by WOMAC scores, was not related significantly to changes in BME lesions [5]. However, in the studies by Felson et al. [2, 7], BME was associated with progressive radiographic knee $\mathrm{OA}$ and pain. Cross sectional associations between BME lesions and clinical findings remain controversial [2-6]. Hence, a lack of clarity about the relationship between changes in BME lesions and WOMAC scores is not surprising. Phan et al. [5] have suggested that the complexity of pain physiology and the difficulty of pain evaluation may explain these findings, as well as the fact that patients experience pain differently. Another important factor might be the stage of $\mathrm{OA}$ in the patients being studied. For instance, pain might be associated with BME lesions in a more developed stage of the disease and less so earlier on. Knee OA studied in the population by Felson [2] was more advanced than the GARP population [8]. Also Felson's population consisted of patients with knee OA only, whereas the GARP 
population is focused on patients with familial OA at multiple sites. A considerable proportion of patients in the present study did not have symptomatic radiographic knee $\mathrm{OA}$ in the imaged knee, and consequently average WOMAC scores in the present population were low (Table 1). Nevertheless, associations between BME lesions and clinical findings are controversial and BME lesions may be excluded ultimately as a factor in pain sensation.

The present study has a number of limitations. Firstly, not all patients with a complete MRI follow-up completed the WOMAC signal knee. Secondly, the inclusion of patients who were first-degree relatives may have introduced an artefact. However, linear regression analysis, with robust standard errors that clustered on pairs, excluded this possibility. Thirdly, although referred pain from the hip may have been a confounder, hip OA occurred in only $7 \%$ of the patients and was not thought a contributory factor.
Fourthly, 2 years of follow-up may be too short for an early OA population. However, we did find a considerable change in size of both cystic and BME lesions. Finally, the term BME used in this article probably should be described more properly as "ill defined signal intensity abnormalities", as the so-called BME pattern in OA knees represents on histological examination nonspecific abnormalities such as bone marrow necrosis, bone marrow fibrosis, trabecular abnormalities and only a small amount of bone marrow oedema [1]. Nevertheless, the term BME is a commonly accepted identity and widely used in the OA literature [2].

In conclusion, BME lesions are shown to be a variable parameter when followed over time in patients with knee $\mathrm{OA}$ and are not predictive of pain.

Acknowledgments This study was supported by Pfizer, Groton, Conn., USA.

\section{References}

1. Zanetti M, Bruder E, Romero J, Hodler $\mathrm{J}$ (2000) Bone marrow edema pattern in osteoarthritic knees: correlation between MR imaging and histologic findings. Radiology 215(3):835-840

2. Felson DT, Chaisson CE, Hill CL, Totterman SM, Gale ME, Skinner KM et al (2001) The association of bone marrow lesions with pain in knee osteoarthritis. Ann Intern Med 134(7):541-549

3. Cicuttini F, Wluka A, Hankin J, Wang Y (2004) Longitudinal study of the relationship between knee angle and tibiofemoral cartilage volume in subjects with knee osteoarthritis. Rheumatology (Oxford) 43(3):321-324

4. Link TM, Steinbach LS, Ghosh S, Ries M, Lu Y, Lane N et al (2003) Osteoarthritis: MR imaging findings in different stages of disease and correlation with clinical findings. Radiology 226(2):373-381
5. Phan CM, Link TM, Blumenkrantz G, Dunn TC, Ries MD, Steinbach LS et al (2006) MR imaging findings in the follow-up of patients with different stages of knee osteoarthritis and the correlation with clinical symptoms. Eur Radiol 16(3):608-618

6. Kornaat PR, Bloem JL, Ceulemans RY, Riyazi N, Rosendaal FR, Nelissen RG et al (2006) Osteoarthritis of the knee: association between clinical features and MR imaging findings. Radiology 239(3):811-817

7. Felson DT, McLaughlin S, Goggins J, LaValley MP, Gale ME, Totterman S et al (2003) Bone marrow edema and its relation to progression of knee osteoarthritis. Ann Intern Med 139(5 Pt 1): 330-336

8. Riyazi N, Meulenbelt I, Kroon HM, Ronday KH, Hellio le Graverand MP, Rosendaal FR et al (2005) Evidence for familial aggregation of hand, hip, and spine but not knee osteoarthritis in siblings with multiple joint involvement: the GARP study. Ann Rheum Dis 64(3):438-443

9. Kellgren JH, Lawrence RC (1957) Radiographic assessment of osteoarthritis. Ann Rheum Dis 16:494-502
10. Bellamy N, Buchanan WW, Goldsmith CH, Campbell J, Stitt LW (1988) Validation study of WOMAC: a health status instrument for measuring clinically important patient relevant outcomes to antirheumatic drug therapy in patients with osteoarthritis of the hip or knee. J Rheumatol 15(12):1833-1840

11. Kornaat PR, Ceulemans RY, Kroon HM, Riyazi N, Kloppenburg M, Carter WO et al (2005) MRI assessment of knee osteoarthritis: Knee Osteoarthritis Scoring System (KOSS)-inter-observer and intra-observer reproducibility of a compartment-based scoring system. Skeletal Radiol 34(2):95-102

12. Mink JH, Deutsch AL (1989) Occult cartilage and bone injuries of the knee: detection, classification, and assessment with MR imaging. Radiology 170 (3 Pt 1):823-829

13. Carrino JA, Blum J, Parellada JA, Schweitzer ME, Morrison WB (2006) MRI of bone marrow edema-like signal in the pathogenesis of subchondral cysts. Osteoarthr Cartil 14(10):1081-1085 